\title{
DETERMINATION OF THE DRAG COEFFICIENT BY ANALYSING THE TRAJECTORY OF A FOOTBALL
}

\author{
Gábor Pszota \\ Associate professor, University of Miskolc, Institute of Physics and Electrical Engineering \\ 3515 Miskolc, Miskolc-Egyetemváros, e-mail: fizpszo@uni-miskolc.hu
}

\begin{abstract}
Two different types of motion were analysed based on video footage using the free software tool named Tracker. One of them involved dropping the football vertically without any spin, the other type was the projectile motion resulted from a goalie punt. The analysis consisted of fitting the time dependence of the coordinates using a sixth order polynomial, then using these functions and other parameters to obtain the drag coefficient as a function of speed and Reynolds number. Similarly to previous works, the drag coefficient showed large differences for the different speeds. The irregularities and asymmetry of the ball also caused the results to be different for the different trials depending on the orientation of the ball, as well as the slight horizontal spin of the ball during its projectile motion. This method can prove to be a useful tool for further studies in a more controlled environment with higher quality new balls.
\end{abstract}

Keywords: drag coefficient; trajectory analysis; projectile motion; drag crisis

\section{Introduction}

When an object travels through the air, it is affected by two things: gravity and the air. Accordingly, the net force acting on the object is the vector sum of the two corresponding forces. The force of gravity is always directed vertically downward with $m g$ magnitude, but the force exerted by the air is rather complicated. Because of this, the assumption that this force is simply proportional to the square of the velocity and directed opposite to the direction of motion [1] is an oversimplification of the problem.

With the exception of American football and rugby, the balls that are used in the different sports are more or less spherical with various surface patterns and roughness. Several reports have been published on the aerodynamic properties of golf balls [2-5], tennis balls [6-8], baseballs [9, 10], cricket balls [11], volleyballs [12], and rugby balls [13, 14].

Various methods have been applied in order to determine the aerodynamic properties of footballs (soccer balls) as well. In these studies the force exerted by the air is broken into three components. The drag is the component that is opposite to the motion of the ball. The component that is perpendicular to the velocity but within the plane formed by the velocity and the weight is called lift, while the other component that is perpendicular to this vertical plane, i.e. it is horizontal, is called sideways force. This sideways force is responsible for the ball leaving the plane formed by the velocity and the weight, and it plays an important role in case of a curve ball. These perpendicular components are caused by the Magnus-effect, i.e. as the spinning ball moves through the air it experiences a force perpendicular to its velocity and the spin axis (horizontal spin axis means topspin or backspin causing lift, vertical spin axis means sideways spin causing sideways force). When the ball does not move because the experiment is conducted in a wind tunnel, the drag is opposite to the velocity of the flow, lift is the vertical component, and the sideways force is the horizontal component that is perpendicular to the wind velocity. The three components are usually written as: 


$$
\begin{aligned}
& F_{D}=\frac{1}{2} \rho A C_{D} v^{2} \\
& F_{L}=\frac{1}{2} \rho A C_{L} v^{2} \\
& F_{S}=\frac{1}{2} \rho A C_{S} v^{2},
\end{aligned}
$$

where $\rho$ is the density of the air, $A$ is the cross-section of the ball, $C_{D}, C_{L}$, and $C_{S}$ are the coefficients for the drag, lift, and sideways force, respectively. Finally, $v$ is the velocity of the air in case of a wind tunnel experiment, or the velocity of the ball in case of trajectory analysis. The unpredictable motion of the football during a shot $[15,16]$ is caused by the fact that these coefficients do not remain constant as the speed of the ball changes.

Several authors [17-21] have reported a sudden drop in the drag coefficient, referred to as drag crisis, as the velocity reaches a critical value. This translates to a critical Reynolds number at which the flow around the ball changes from laminar to turbulent. The Reynolds number is proportional to the velocity:

$$
\operatorname{Re}=\frac{\rho v d}{\mu},
$$

where $\rho$ is the density of the air, $v$ is the velocity of the air or the ball, $d$ is the diameter of the ball, and $\mu$ is the dynamic viscosity of the air. Above this critical velocity the drag coefficient seems to increase linearly [22].

In order to quickly summarize the main methods and results of the literature related to football, let's start with the work of Asai et al. [15], in which they explained the results of previous studies through the example of the famous free kick taken by Roberto Carlos against France in 1998. One important point of the article is that the laminar to turbulent transition happens at smaller velocities if the surface is rough, therefore in case of a football this means smaller critical velocities compared to a smooth sphere. This can result in the drag coefficient dropping from about 0.5 to as low as 0.15 at velocities that depend on the pattern of the football. The other important point in the article was that for a spinning ball the coefficients of the lateral forces increase as the ball slows down; and this explains the sudden curving of the mentioned free kick along the final meters.

Carré et al. [23] analysed the trajectories of footballs launched at different velocities and spin rates. The raw data for the $x$ and $y$ coordinates were fitted using quadratic polynomials for the observed almost 0.7 seconds. These fits were compared with a numerical simulation to determine the drag and lift coefficients, which were taken to be constants for each trial. In case of a non-spinning ball they determined that the drag coefficient grew linearly from about 0.05 to 0.35 as the velocity increased from $15 \mathrm{~m} / \mathrm{s}$ to $35 \mathrm{~m} / \mathrm{s}$. The lift coefficient was very close to zero with an average of 0.02 . This small number could still be enough to cause an unpredictable flight path for a non-spinning football, and it is probably the result of the asymmetries of the shape and surface pattern as discussed in [16]. The other part of this work investigated the effect of spin on the drag and lift coefficients, and it showed that they both increase with larger spin if the velocity remains the same.

Asai et al. [17] used wind tunnel experiments to measure the drag, lift, and sideways coefficients for different velocities in case of non-rotating and rotating balls, as well as a smooth sphere. For the three different non-rotating balls the wind speed was changed from $7 \mathrm{~m} / \mathrm{s}$ to $35 \mathrm{~m} / \mathrm{s}$, and the drag crisis was 
observed for the different footballs at Re values of $\sim 2.2 \times 10^{5}$ and $\sim 3.1 \times 10^{5}$, corresponding to wind speeds of about $15 \mathrm{~m} / \mathrm{s}$ and $22 \mathrm{~m} / \mathrm{s}$.

Oggiano and Saetran [21] performed wind tunnel experiments and trajectory simulation with three different modern match balls and a replica for wind speeds between $6 \mathrm{~m} / \mathrm{s}$ and $26 \mathrm{~m} / \mathrm{s}$. The critical Re values for non-spinning balls were found to be in the $1.2-2 \times 10^{5}$ range, corresponding to $8.5-14 \mathrm{~m} / \mathrm{s}$ wind speeds.

Alam et al. [18] compared 32 panel and 14 panel footballs during wind tunnel experiments for wind speeds between $5.6 \mathrm{~m} / \mathrm{s}$ and $36 \mathrm{~m} / \mathrm{s}$. To understand the airflow around the ball better, they visualized it using smoke. They showed that the 14 panel football is closer to the smooth sphere in terms of its behaviour than the 32 panel football, meaning that the transition to turbulent flow happens at higher wind speeds. Because of this the 14 panel ball will potentially experience more drag at lower speeds than the 32 panel ball. The transition happened in the Re range of $1.1-3 \times 10^{5}$, corresponding to wind speeds between $7.7 \mathrm{~m} / \mathrm{s}$ and $21 \mathrm{~m} / \mathrm{s}$. Above $17 \mathrm{~m} / \mathrm{s}$ however, the drag coefficient remained more or less constant with increasing wind speeds, and the average was around 0.23 for all the balls.

Alam et al. [20] evaluated the aerodynamic properties of three different footballs and a smooth sphere. One of the balls was the Adidas Jabulani, which was famous for its unpredictable behaviour in the air. They used wind tunnel experiments to measure the drag, lift, and sideways components at wind velocities between $8.3 \mathrm{~m} / \mathrm{s}$ and $36 \mathrm{~m} / \mathrm{s}$. The airflow became fully turbulent at $8.3 \mathrm{~m} / \mathrm{s}\left(\operatorname{Re}=1.1 \times 10^{5}\right)$, $11.1 \mathrm{~m} / \mathrm{s}\left(\operatorname{Re}=1.6 \times 10^{5}\right)$, and $16.7 \mathrm{~m} / \mathrm{s}\left(\operatorname{Re}=2.4 \times 10^{5}\right)$ for the three different balls, while the result for the smooth sphere was similar to previous studies [24] at $27.8 \mathrm{~m} / \mathrm{s}\left(\mathrm{Re}=3.7 \times 10^{5}\right)$. Another interesting result of this work was that they measured the difference in the drag coefficient depending on which side of the ball was facing the wind. This difference turned out to be 8-9\% in case of the Jabulani ball and less than $2 \%$ in case of the traditional 32 panel ball. This explains the unpredictable behaviour of the Jabulani ball even without any spin.

Goff and Carré [23] used a ball launcher and high speed cameras to analyse the trajectory of different balls launched at different velocities and with different spin rates. For non-spinning balls they analysed trajectories with velocities in the $15.6 \mathrm{~m} / \mathrm{s}-19.3 \mathrm{~m} / \mathrm{s}$ range in order to remain above the transition region, that way they could assume that the drag coefficient stayed constant. They found that the value was 0.17 , which was consistent with the wind tunnel experiments. Also, by launching the footballs through a dust cloud they could also examine the separation of the boundary layer from the football.

Kray et al. [19] measured the Magnus-effect on a prototype model football that was rotated perpendicular to the flow direction. They confirmed that the subcritical drag coefficient was about 0.5 for a stationary ball, and that the wind speed at which the transition from laminar to turbulent flow occurs depends on the surface roughness of the ball, in agreement with previous works [25]. Using aerosol technique they also managed to visualize the flow for both a stationary and a rotating model ball.

\section{Trajectory analysis}

Studying the footballs in flight as opposed to conducting wind tunnel experiments has the advantage that the ball is free to move in the air. It may be true that mounting the ball onto a support rod allows for a more controlled environment during the wind tunnel experiment, but at the same time the aerodynamic effect of the support rod will always influence the results to some extent. In the literature there are some experiments where the authors used trajectory analysis one way or another. The approach followed by Carré et al. [22] resulted one pair of values for the drag and lift coefficients during each trial even though 
the recording took almost 0.7 seconds, during which the velocity of the ball must have varied significantly. The ball projection machine didn't make it possible to control the orientation of the ball seams, therefore they used random orientation. One can also wonder if the drag and lift are affecting the ball during the flight, how valid is the assumption to fit the $x$ and $y$ coordinates using quadratic functions. Finally, since they measured increasing drag coefficients as the velocity increased from $15 \mathrm{~m} / \mathrm{s}$ to 35 $\mathrm{m} / \mathrm{s}$, it seems that all of the measurements were performed above the critical velocity. Ten years later Goff and Carré [23] investigated the drag and lift force components for balls that had pure topspin or backspin. They again treated the drag and lift coefficients as constants for each trial, and they justified this assumption by keeping the velocities above the critical velocity at all times. In their earlier paper [26] where they discussed their numerical model for obtaining the coefficients, they even used the range of the flight to obtain the drag coefficient for non-spinning balls. This would assume that the coefficient remained the same during the whole flight for the various velocity values. Again, this is justified by the authors based on keeping the speed of the ball above the critical limit for the whole flight. This might still be an oversimplification, since the drag coefficient could vary with the speed even beyond the critical value.

In the present paper the trajectories for non-spinning or horizontally slowly spinning footballs were analysed in such a way that the value of the drag coefficient could be obtained in each point, i.e. in each moment. It is certainly true that using the raw data to numerically obtain the acceleration can result in unrealistic fluctuations because of the small errors in the position data. To circumvent this issue the position data were fitted using sixth degree polynomials to smooth out the graphs and average out any potential errors in marking the position of the ball on the screen. Once the polynomial function was obtained for the position, the first derivative was taken to derive the velocity data, and the second derivative gave the acceleration data. Two different types of motion were analysed as seen in Figure 1 and 2. In one case the ball was simply dropped from the top of a ten-storey building without any spin, while in the other case a goalie punt was performed and the resulting projectile motion was analysed. In all cases the videos were recorded in $1080 \mathrm{p}$ resolution at 30 frames per second. This means having a data point every 0.033 seconds.

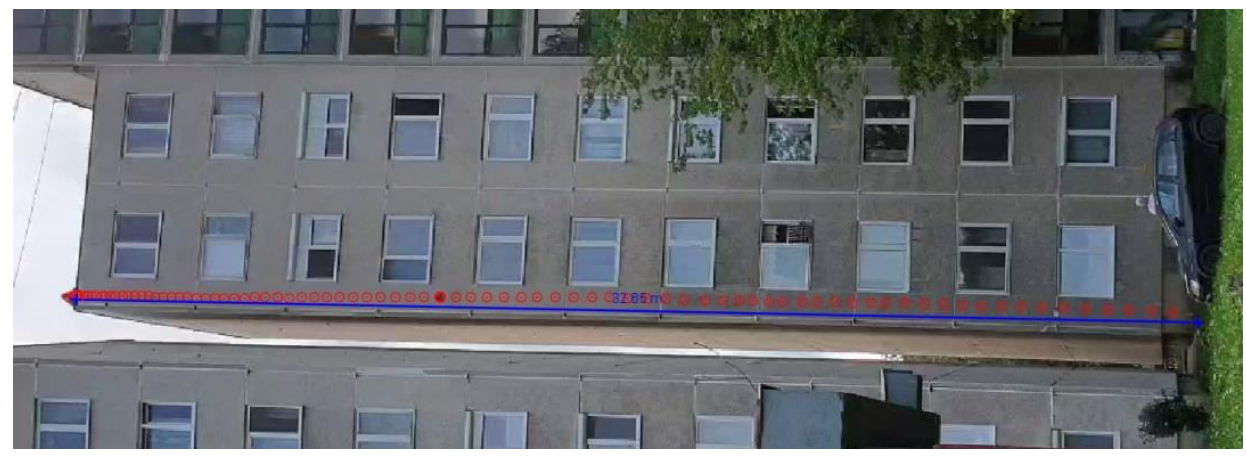

Figure 1. Trajectory of the ball that was dropped from the ten-storey building

To analyse the motion of the football based on the videos the free software tool named Tracker [27] was used. The program was downloaded from the Open Source Physics [28] website. After obtaining and exporting the coordinate data for the different trials, they were imported into Excel, where the fitting was performed. The sixth degree polynomials were chosen in each case because that was the highest degree available. In some of the cases lower order polynomials could have also given satisfactory fit to the data, but the same type of functions were selected in each case. 


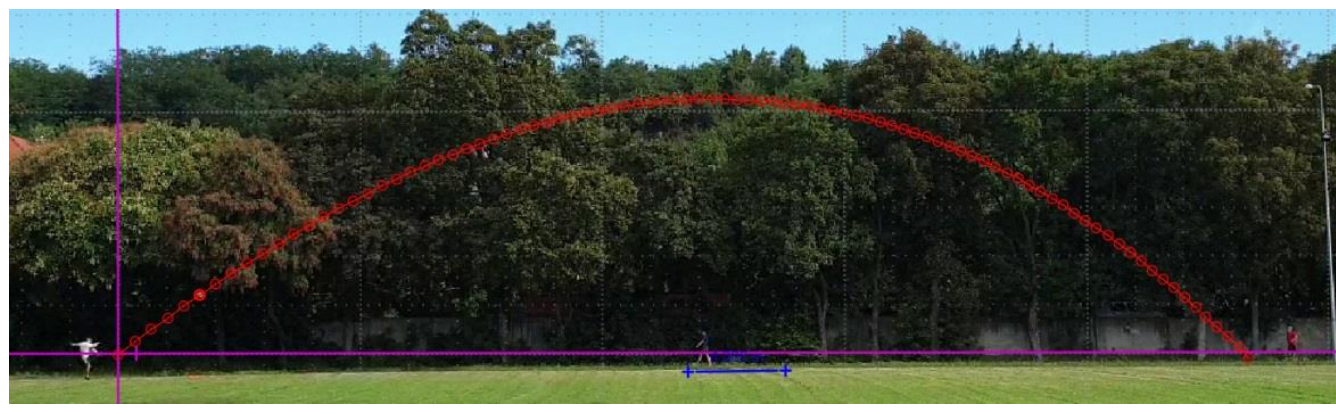

Figure 2. Trajectory of the ball for one of the goalie punts

An example for the fitting procedure is presented in Figure 3 and 4. Three drops were performed, but during one of the drops the knuckle-effect caused the ball to deviate from the vertical direction and it hit the side of the building. This effect can also be seen to some extent in Figure 1. Out of the several goalie punts only four were used, because it was hard to hit the ball in such a way that it flew in the predetermined plane without any topspin or backspin. A slight horizontal spin was noticeable during the flight for some of the kicks by watching the ball in the air.

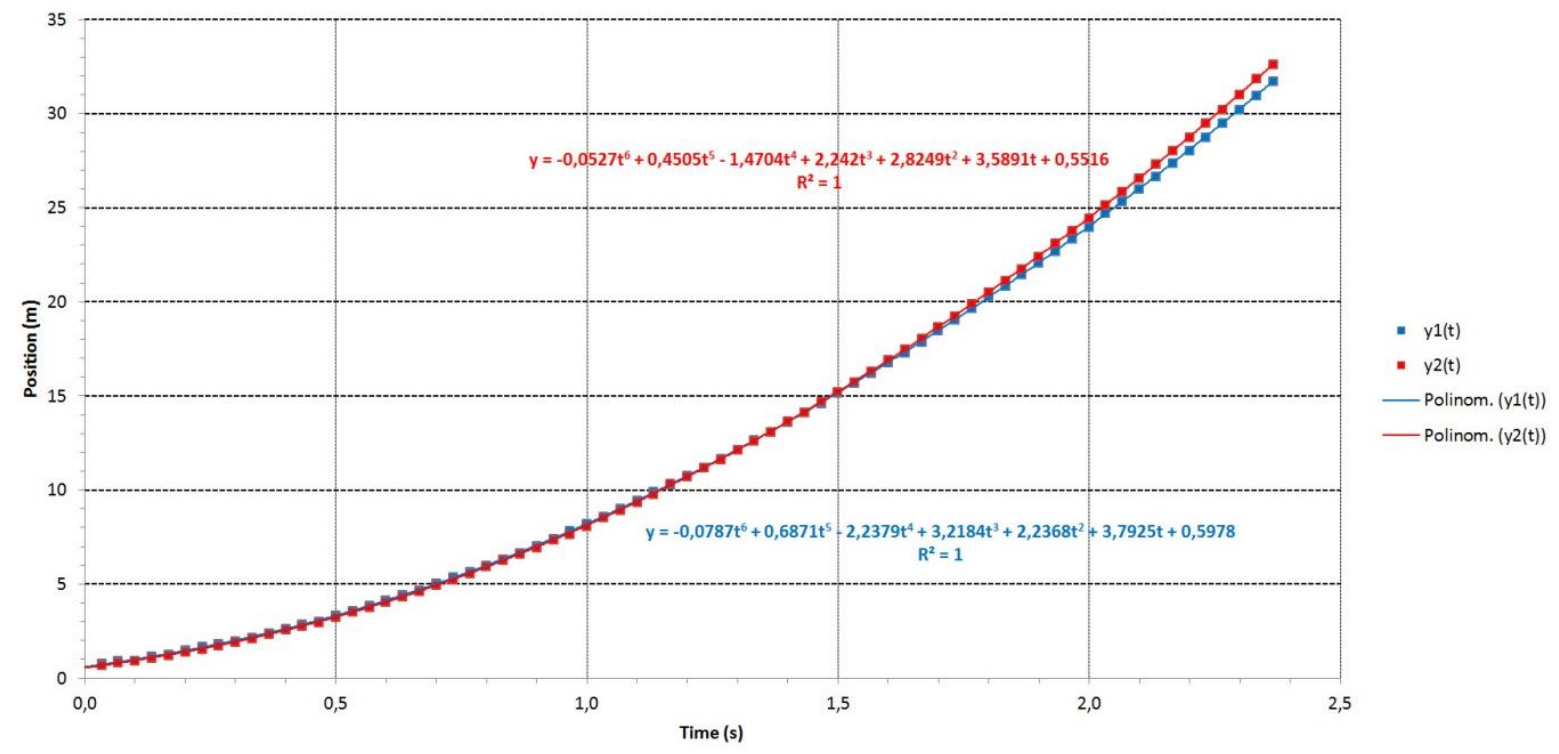

Figure 3. Fitting the position vs. time graphs for the two drops

Considering that for all the trials the spin of the ball was zero or negligible, the Magnus-effect could be ignored, and the coefficients of the lift and the sideways force were taken to be zero. This means that in this work the force exerted by the air was taken to be exactly opposite to the direction of the velocity and only the coefficient of the drag component was examined. The previously mentioned slight knuckleeffect that can be noticed for a non-spinning ball was ignored during the analysis. The experiments were conducted in nice sunny weather, the temperature was about $23^{\circ} \mathrm{C}$, the relative humidity was about $58 \%$, and the atmospheric pressure was about $101100 \mathrm{~Pa}$.

The value for the dynamic viscosity was interpolated based on the listed values at the engineeringtoolbox.com website, and the $\mu=18.27 \times 10^{-6} \mathrm{~Pa} \cdot \mathrm{s}$ value was used. The density for the mentioned 
atmospheric conditions was obtained from the omnicalculator.com website, and it turned out to be $\rho=1.182 \mathrm{~kg} / \mathrm{m}^{3}$. Using these values the connection between the speed and the Reynolds number is the following:

$$
\operatorname{Re}=14233 \frac{\mathrm{s}}{\mathrm{m}} \cdot v
$$

The other parameters needed for the analysis were the mass of the ball $(m=0.438 \mathrm{~kg})$, its diameter $(d=220 \mathrm{~mm})$, and the acceleration due to gravity $\left(g=9.807 \mathrm{~m} / \mathrm{s}^{2}\right)$. Therefore the cross sectional area of the size 5 ball was $A=0.038 \mathrm{~m}^{2}$.

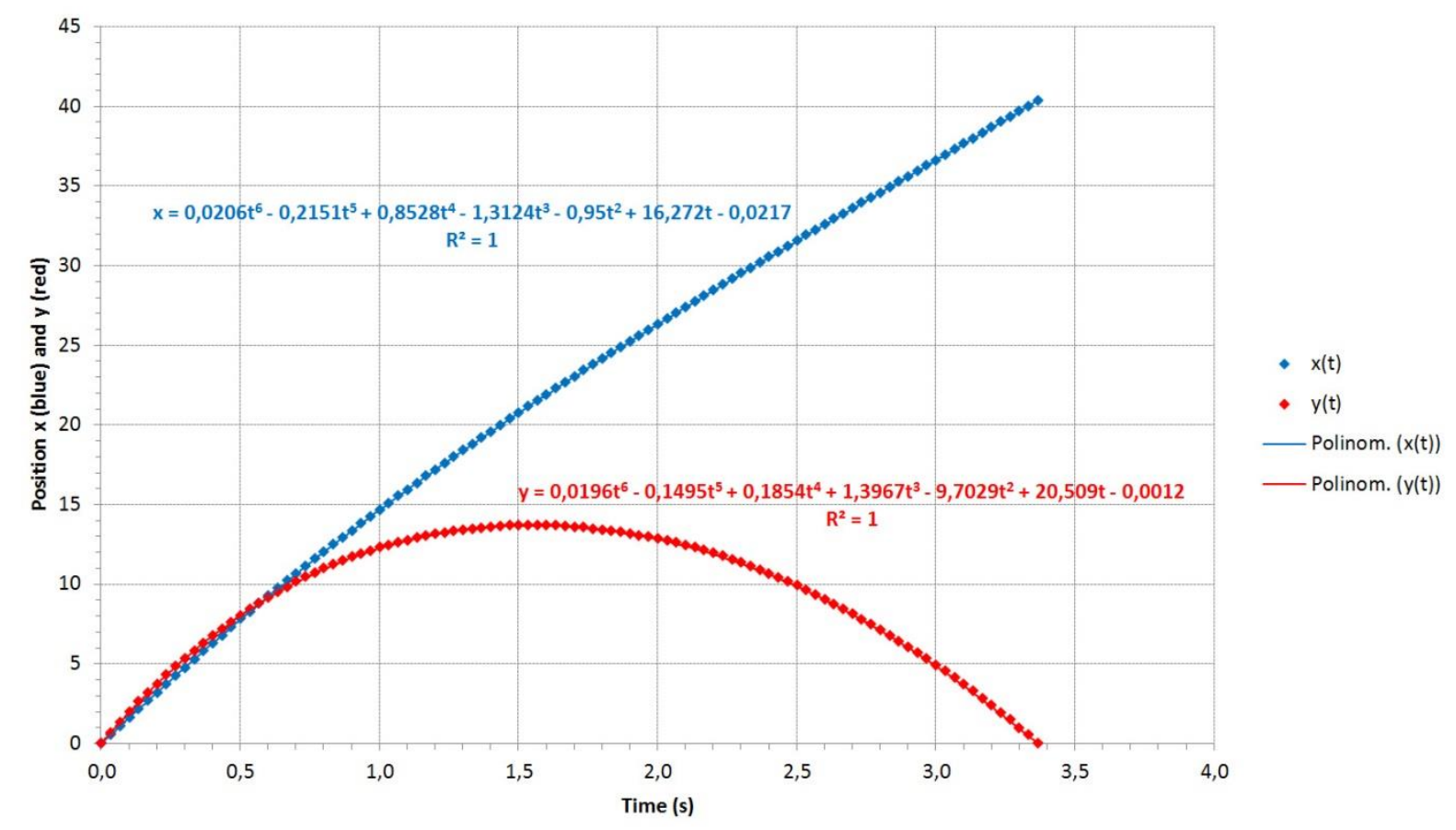

Figure 4. Fitting the $x$ and $y$ position data for one of the goalie punts

The type of ball used was a Sondico Fusion Hybrid technology FIFA football. It was not a new or high quality ball; therefore some asymmetries in the surface pattern and shape were apparent. The ball was simply placed on a kitchen scale at the location of the experiment; therefore the buoyant force exerted by the air was included in the measurement. This was calculated to be about $0.065 \mathrm{~N}$ for such a size 5 ball, which makes the real mass of the ball that multiplies the acceleration in the equation of motion larger by about $1.5 \%$ compared to the measured value. On the other side of the equation, the effect of buoyancy is naturally included in the measured weight. This little discrepancy was ignored during the calculations, similarly to earlier works by other authors [23].

\section{Results of the dropping trials}

The football was started from the top of the building without any initial speed or spin. The exact height of the building was measured by hanging down a rope with a weight, and this value of 32.65 meters was 
used for the calibration of the video. The forces acting on the ball during the fall are in opposite direction, gravity with $m g$ magnitude is pointing down in the direction of the velocity, while the drag is pointing up. The knuckle-effect was ignored, and the motion was treated to be one-dimensional, using only the data of one coordinate. This seems reasonable in the absence of spin, since any horizontal displacement or velocity component is much smaller than the vertical component. Therefore by pointing the $x$ axis downward, the equation of motion can be written as:

$$
m a=m g-\frac{1}{2} \rho C_{D} A v^{2} .
$$

From this equation the drag coefficient can be expressed as a function of the speed and acceleration. The results of the two trials are summarized in Figure 5 and 6. It can be seen from the graphs that the drag coefficient decreases until about $9 \mathrm{~m} / \mathrm{s}$ in both cases, corresponding to about 130000 in terms of Reynolds number, but then it behaves differently for the two trials. Finally, the beginning of an increase can be seen in both graphs above $22 \mathrm{~m} / \mathrm{s}$ or 300000 Re value.

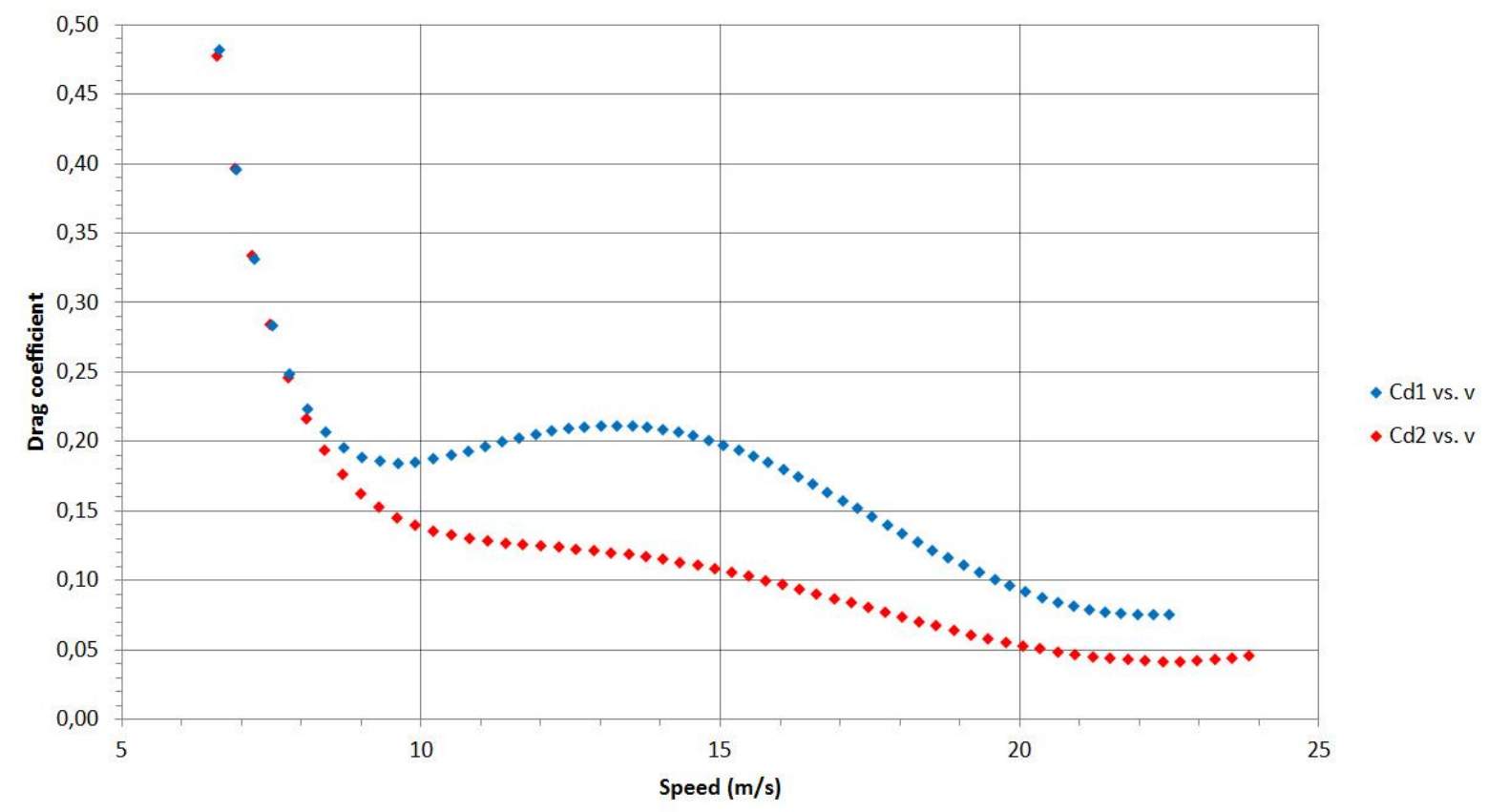

Figure 5. Drag coefficient as a function of the speed 


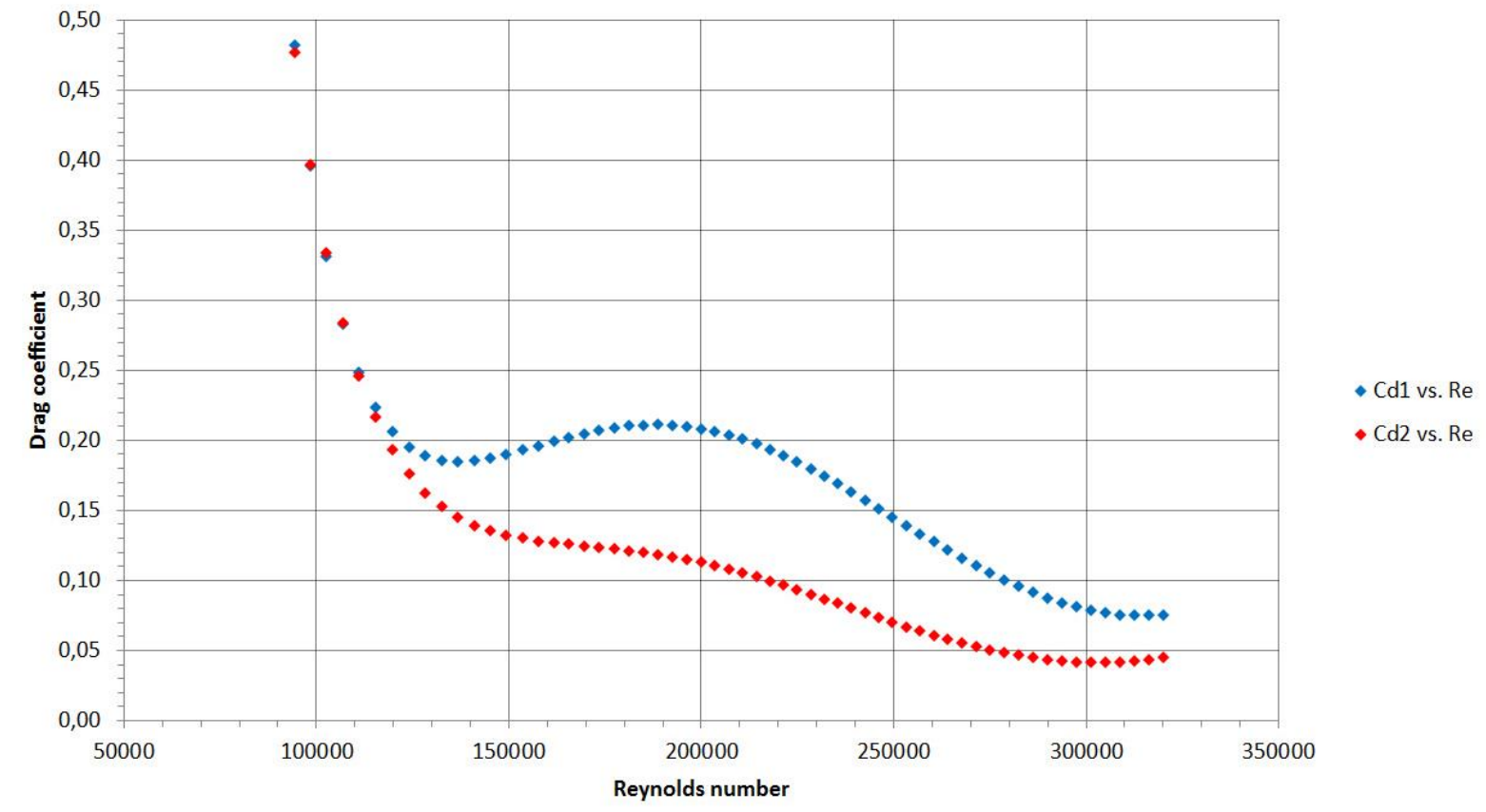

Figure 6. Drag coefficient as a function of the Reynolds number

The first few metres of the fall weren't considered for the fit because of the relatively large errors compared to the small displacements, and even then, the obtained coefficient values were way too large to be accurate. Therefore only those points were graphed where the speed of the football exceeded 6 $\mathrm{m} / \mathrm{s}$. The difference in the two trials could have been caused by starting the ball with different orientation, as well as by the unpredictable effects that arose due to the asymmetries.

\section{Results of the goalie punts}

During the projectile motion of the non-spinning football, the relative directions of the forces are shown in Figure 7. In this case the video was calibrated by placing down two cones 4 meters apart along the flight of the ball.
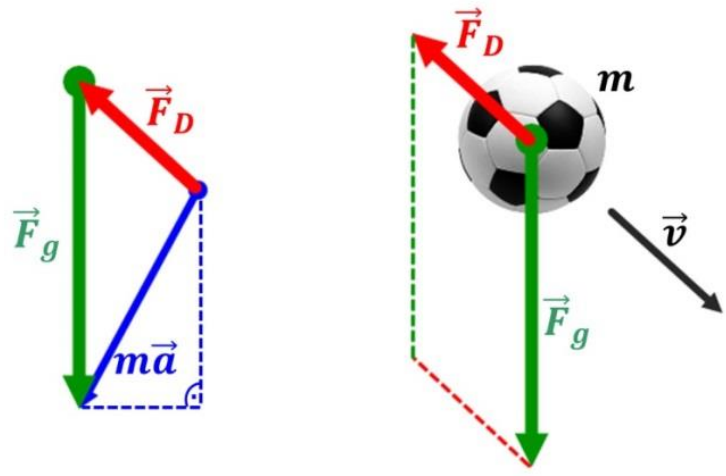

Figure 7. Relative orientation of the forces and the velocity during a projectile motion 

form:

This is now a two-dimensional problem, therefore the equation of motion has to be written in vector

$$
m \vec{a}=\vec{F}_{g}+\vec{F}_{D}
$$

Rearranging this equation gives the following result for the magnitude of the drag:

$$
F_{D}=\frac{1}{2} \rho C_{D} A v^{2}=\left|m \vec{a}-\vec{F}_{g}\right|=|m \vec{a}+m g \vec{j}|,
$$

where $\vec{j}$ is the basis vector along the $y$ axis, directed upward. This gives the following expression for the drag coefficient in terms of the obtained velocity and acceleration components:

$$
C_{D}=\frac{2 m}{\rho A v^{2}}|\vec{a}+g \vec{j}|=\frac{2 m}{\rho A\left(v_{x}^{2}+v_{y}^{2}\right)} \sqrt{a_{x}^{2}+\left(a_{y}+g\right)^{2}} .
$$

The drag coefficient values calculated for the four different trials are summarized in Figure 8 and Figure 9. As it is obvious from the graphs, this approach has resulted very different values for the drag coefficient for the different velocities, not just if the different trials are compared to each other, but even within one trial. It seems that the coefficient had one value for the rising part of the projectile motion for a certain speed, and then it took a different value at the same speed when the ball was going downward. Even the high speed region of the graph is not decisive in term of the coefficient growing larger or decreasing, i.e. whether the speed is still in the transition region or above that.

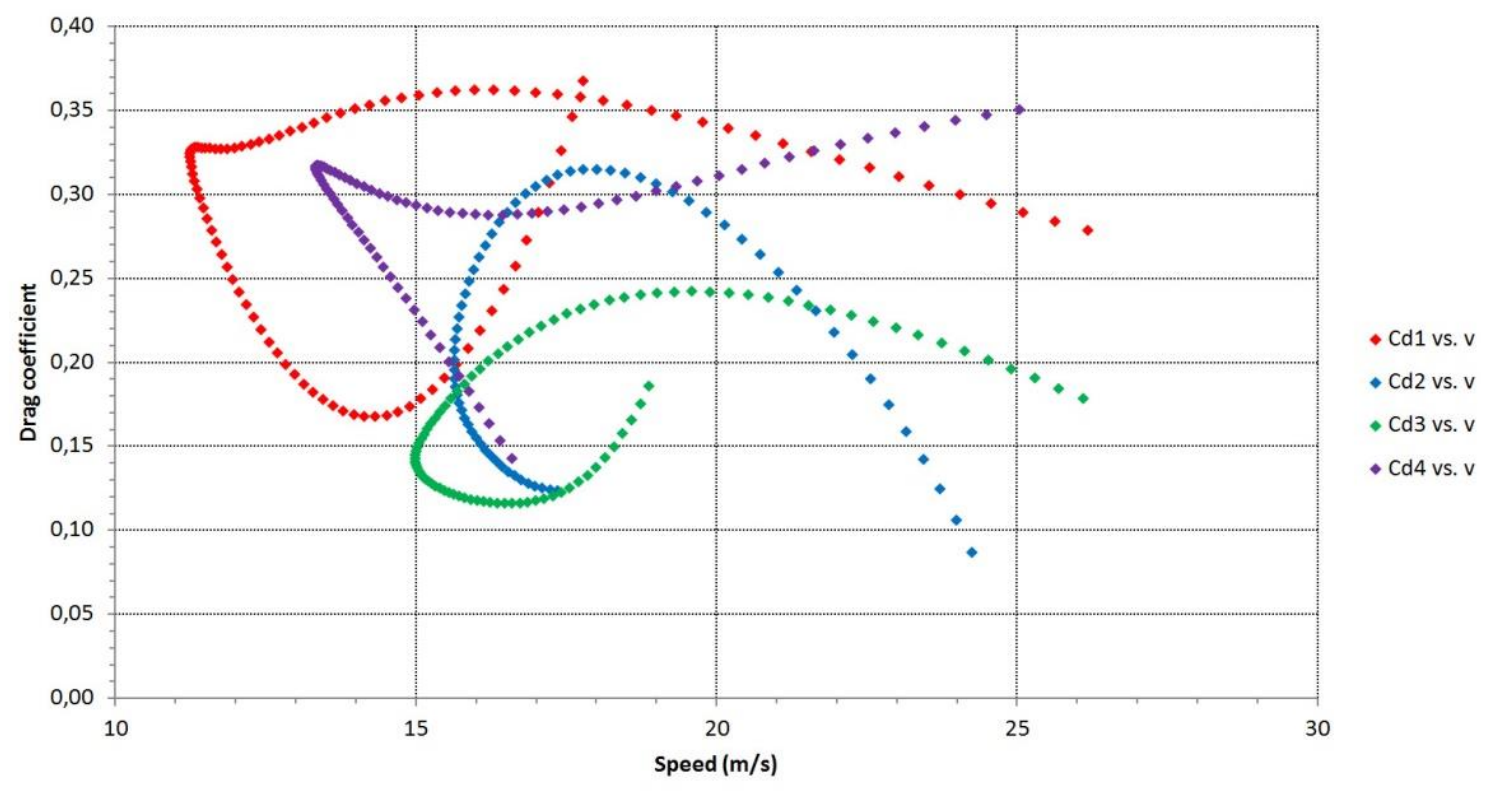

Figure 8. Drag coefficient as a function of the speed during the four trials of goalie punt 


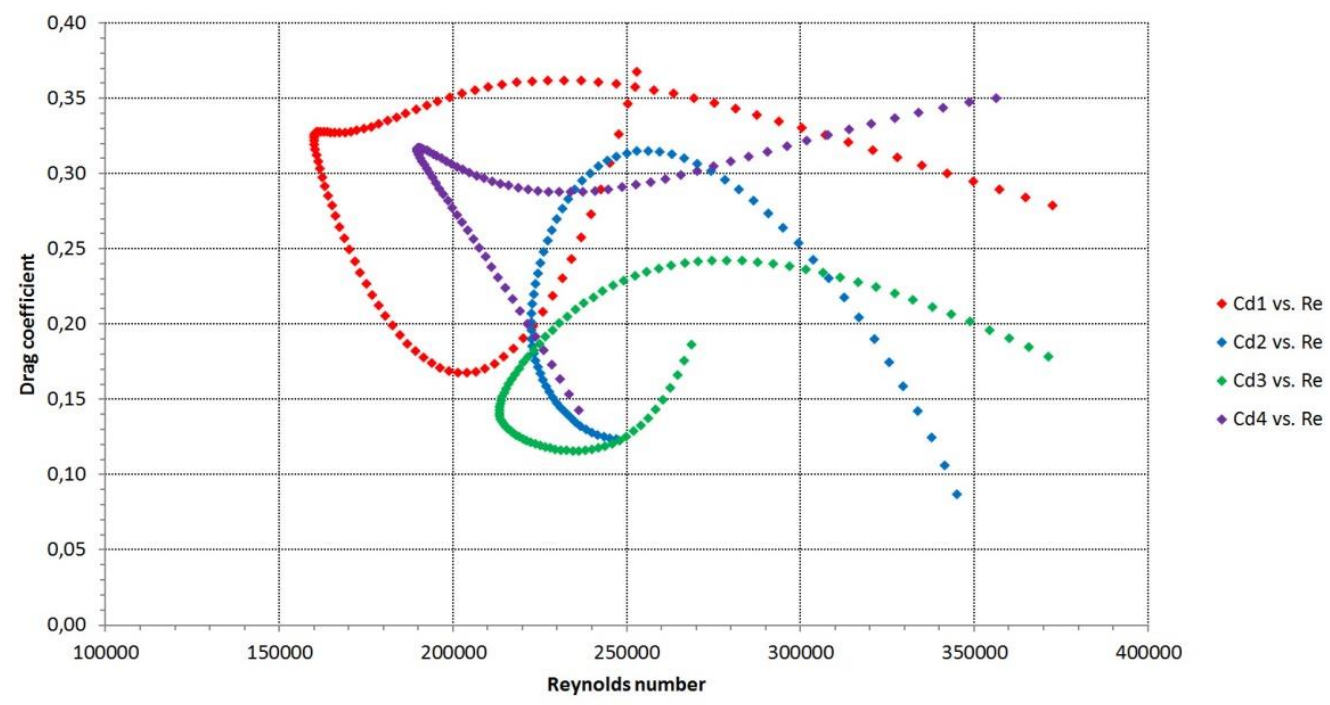

Figure 9. Drag coefficient as a function of the Reynolds number during the four trials of goalie punt

\section{Summary}

In this paper two new approaches were examined for the study of the drag coefficient by performing trajectory analysis. One approach involved dropping a ball from the top of a ten-storey building without any initial speed or spin. This approach seems to have some merit, since the ball can be started in such a controlled way, and all the different speeds can be investigated during the fall. The orientation of the ball before the drop does have an effect on the results, as it can be seen in the graphs. This orientation can even change during the fall as the asymmetry of the ball causes some knuckle-effect. Therefore this method, just like any method involving trajectory analysis, is not suitable for experiments where the orientation has to be kept fixed. It is also fair to speculate that the wind conditions could have changed along the way as the ball was going downward, since the experiment was conducted outdoors. The other approach during which the projectile motion of the ball was analysed gave very different results for the different trials, and the coefficient even depended on whether the ball was in the rising or descending phase of the motion. The fact that the experiment was conducted outdoors could have played a role in the discrepancies here as well. Based on the results and experiences, this method can be improved in many ways for both approaches. First of all, the experiment should be conducted indoors, using brand new high quality balls. With the current apparatus the frame rate can be increased to 60 frames per second at 1080p resolution, or even higher if the resolution is decreased. In this case, however, it is probably not possible to record a 32 meter flight and still see the football well in the video. The solution could be to record only a certain part of the trajectory with one camera. The second approach could probably be improved greatly by having a controlled way of launching the ball instead of just kicking it.

\section{Acknowledgement}

The research reported here was carried out as part of the EFOP-3.6.1-16-2016-00011 "Younger and Renewing University - Innovative Knowledge City - Institutional development of the University of 
Miskolc aiming at intelligent specialization" project implemented in the framework of the Szechenyi 2020 program. The realization of this project is supported by the European Union, co-financed by the European Social Fund.

\section{References}

[1] Chudinov, P., Eltyshev, V. and Barykin, Y.: Analytical construction of the projectile motion trajectory in midair, (2020). arXiv:2007.14991

https://archive.org/details/principleofrelat00eins/page/n33

[2] Thompson, J. J.: The dynamics of a golf ball, Nature, 85, (1910), 2151-2157.

[3] Davies, J. M.: The aerodynamics of golf balls, Journal of Applied Physics, 20, (1949), 821-828. https://doi.org/10.1063/1.1698540

[4] Bearman, P. W., Harvey, J. K.: Golf ball aerodynamics, Aeronautical Quarterly, 27, (1976), 112122. https://doi.org/10.1017/S0001925900007617

[5] Smits, A. J., Ogg, S.: Aerodynamics of golf ball, in Hung, G. K., Pallis, J. M. (eds) Biomedical Engineering Principles in Sports, (2004), pp. 333-364. Kluwer Academic Plenum Publishers, New York

[6] Stepanek, A.: The aerodynamics of tennis balls - the topspin lob, American Journal of Physics, 56, (1988), 138-142. https://doi.org/10.1119/1.15692

[7] Chadwick, S. G., Haake, S. J.: The drag coefficient of tennis balls, in Subic, A., Haake, S. J. (eds) The Engineering of Sport, (2000), pp. 169-176. Blackwell Science, Oxford

[8] Haake, S. J., Chadwick, S. G., Dignall, R. J., Goodwill, S. R., Rose, P.: Engineering tennis slowing the game down, Sports Engineering, 3, (2000), 131-143. https://doi.org/10.1046/j.1460-2687.2000.00040.x

[9] Watts, R. G., Sawyer, E.: Aerodynamics of a knuckleball, American Journal of Physics, 43, (1975), 960-963. https://doi.org/10.1119/1.10020

[10] Watts, R. G., Ferrer, R.: The lateral force on a spinning sphere: aerodynamics of a curveball, American Journal of Physics, 55, (1987), 40-44. https://doi.org/10.1119/1.14969

[11] Mehta, R. D., Bentley, K., Proudlove, M., Varty, P.: Factors affecting cricket ball swing, Nature, 303, (1983), 787-788. https://doi.org/10.1038/303787a0

[12] Wei, Q., Lin, R., Liu, Z.: Vortex-induced dynamics load on a non-spinning volleyball, Fluid Dynamics Research, 3 (1988), 231-237. https://doi.org/10.1016/0169-5983(88)90071-8

[13] Seo, K., Kobayashi, O., Murakami, M.: Regular and irregular motion of a rugby football during flight, in Hubbard, M., Mehta, R. D., Pallis, J. M. (eds) The Engineering of Sport 5, Vol. 1, pp. 567-573. International Sports Engineering Association, Sheffield

[14] Alam, F., Subic, A., Watkins, S., Naser, J., Rasul, M. G.: An experimental and computational study of aerodynamic properties of rugby balls, WSEAS Transactions on Fluid Mechanics, 3, (2008), 279-286.

[15] Asai, T., Akatsuka, T., Haake, S. J.: The physics of football, Physics World, 11, (1998), 25-27. https://doi.org/10.1088/2058-7058/11/6/24

[16] Hong, S., Kazama, Y., Nakayama, M., Asai, T.: Ball impact dynamics of knuckling shot in soccer, Procedia Engineering, 34, (2012), 200-205. https://doi.org/10.1016/j.proeng.2012.04.035

[17] Asai, T., Seo, K., Kobayashi, O., Sakashita, R.: Fundamental aerodynamics of the soccer ball, Sports Engineering, 10, (2007), 101-110. https://doi.org/10.1007/BF02844207

[18] Alam, F., Chowdhury, H., Moria, H., Fuss, F. K.: A comparative study of football aerodynamics, Procedia Engineering, 2, (2010), 2443-2448. https://doi.org/10.1016/j.proeng.2010.04.013 
[19] Kray, T., Franke, J., Frank, W.: Magnus effect on a rotating soccer ball at high Reynolds numbers, Journal of Wind Engineering and Industrial Aerodynamics, 124, (2014), 46-53. https://doi.org/10.1016/j.jweia.2013.10.010

[20] Alam, F., Chowdhury, H., Moria, H., Fuss, F. K., Khan, I., Aldawi, F., Subic, A.: Aerodynamics of contemporary FIFA soccer balls, Procedia Engineering, 13, (2011), 188-193. https://doi.org/10.1016/j.proeng.2011.05.071

[21] Oggiano, L., Saetran, L.: Aerodynamics of modern soccer balls, Procedia Engineering, 2, (2010), 2473-2479. https://doi.org/10.1016/j.proeng.2010.04.018

[22] Carré, M. J., Asai, T., Akatsuka, T., Haake, S. J.: The curve kick of a football II: flight through the air, Sports Engineering, 5, (2002), 193-200.

https://doi.org/10.1046/j.1460-2687.2002.00109.x

[23] Goff, J. E., Carré, M. J.: Investigations into soccer aerodynamics via trajectory analysis and dust experiments, Procedia Engineering, 34, (2012), 158-163. https://doi.org/10.1016/j.proeng.2012.04.028

[24] Achenbach, E.: Experiments on the flow past spheres at very high Reynolds numbers, Journal of Fluid Mechanics, 54(3), (1972), 565-575. https://doi.org/10.1017/S0022112072000874

[25] Achenbach, E.: The effects of surface roughness and tunnel blockage on the flow past spheres, Journal of Fluid Mechanics, 65(1), (1974), 113-125. https://doi.org/10.1017/S0022112074001285

[26] Goff, J. E., Carré, M. J.: Trajectory analysis of a soccer ball, American Journal of Physics, 77, (2009), 1020-1027. https://doi.org/10.1119/1.3197187

[27] Brown, D.: Tracker Video Analysis and Modeling Tool, Open Source Physics comPADRE, (2012), USA. https://www.compadre.org/osp/items/detail.cfm?ID=7365

[28] Christian, W., Esquembre, F., Barbato, L.: Open Source Physics, Science, (2011), Vol. 334, Issue 6059, pp. 1077-1078. DOI: 10.1126/science.1196984 https://doi.org/10.1126/science.1196984 